\title{
X-Ray Absorption Studies of Ge Layers Buried in Silicon Crystal
}

\author{
I.N. DEMCHENKO ${ }^{a, *}$, K. EAWNICZAK-JABŁońSKA ${ }^{a}$, \\ K.S. ZhuravleV ${ }^{b}$, E. Piskorska ${ }^{a}$, A.I. Nikiforov ${ }^{b}$ \\ AND E. WELTER ${ }^{c}$ \\ ${ }^{a}$ Institute of Physics, Polish Academy of Sciences \\ al. Lotników 32/46, 02-668 Warsaw, Poland \\ ${ }^{b}$ Institute of Semiconductor Physics \\ Siberian Branch of Russian Academy of Sciences \\ prospect Lavrentieva 13, Novosibirsk, Russia \\ ${ }^{c}$ HASYLAB at DESY, Notkestrasse 85, 22607 Hamburg, Germany
}

\begin{abstract}
Polarization-dependent X-ray absorption spectroscopy was used to study the local microstructure of Ge layers buried in silicon. The layers with thickness from 6 to 20 monolayers of Ge were grown by molecular beam epitaxy on $\mathrm{Si}$ substrate and were covered by $\mathrm{Si}(20 \mathrm{~nm})$. To investigate the morphology of grown structures, X-ray absorption near edge structure and extended X-ray absorption fine structure analysis of the Ge $K$-edge was done. The performed qualitative analysis proves that X-ray absorption spectra are very sensitive to the local order in the formed structures and are sources of unique information about morphology of the buried Ge layers. Using these techniques we were able to observe the changes in atomic order around the Ge atoms in investigated buried layers and compare the formed atomic order with that in crystalline Ge. A substantial increase in intensity, broadening and chemical shift of the X-ray absorption near edge structure spectrum for $8 \mathrm{ML}$ were observed. It can be related to the increase in density of electron states caused by increase in the localization of the states due to potential appearing at the Ge island boundaries and indicated the formation of quantum dots. The observed in-plane modulations of radial distribution and out-of-plane for different layers were discussed.
\end{abstract}

PACS numbers: 72.80.Ey, 78.70.Dm, 81.05.GC

*corresponding author; e-mail: demch@ifpan.edu.pl 


\section{Introduction}

The physical properties of quantum confined semiconductor heterostructures have attracted wide attention both from the experimental and theoretical point of view because of their potential applications in microelectronics and development of Si-based optoelectronics. Particularly, there is a considerable interest in the improvement of the electron-hole recombination efficiency of indirect-gap semiconductors using quantum low-dimension effects. A major concept of the method leading to self-organizing quantum dots formation consists in the growth of a layer with several monolayers of one semiconductor in a matrix of another semiconductor with highly mismatched lattice parameters. The growth mechanisms are closely related to both self-assembly nanocrystal formation and band engineering. Due to the well known growth technology of Si and Ge crystals and the large lattice mismatch (about 4\%), the Ge/Si systems attract a great attention today with a continuing hope of creating high efficiency GeSi-based optoelectronic devices. Therefore, the mechanisms taking place during the Ge quantum dots formation are of particular interest. The properties of $\mathrm{Si} / \mathrm{Ge} / \mathrm{Si}$-structures are studied most actively by the methods of electron and atomic-force microscopy (AFM) [1-5], photoluminescence [2-8], and Raman scattering [2, 9-11]. Several studies have been reported on the interface structure and growth morphologies of Ge on Si crystal [12-15], but researchers are still far from understanding and controlling the morphology of interface particularly in the buried layers when AFM cannot be applied.

Recognizing the advantages of X-ray absorption spectroscopy as being a local atom probe and giving a fingerprint of chemical bonding, we applied this spectroscopy to study the local arrangement and chemical bonding of Ge monolayers buried in Si exploring also the linear polarization of synchrotron radiation.

\section{Experimental}

The growth of quantum dots containing heterostructures (with in situ RHEED control) was done in "KATUN-S" MBE installations. Each MBE installation was equipped with two electron beam evaporators for germanium and silicon. The Si/Ge/Si structures were grown on (001) Si substrates. The epitaxial Si buffer layer with the thickness $100 \mathrm{~nm}$ was produced at substrate temperature $600^{\circ} \mathrm{C}$. In order to reduce the size of the quantum dots, the temperature of Si buffer was decreased to $210^{\circ} \mathrm{C}$ and a layer of germanium with thickness from 6 to 20 monolayers (ML) was grown. Next the structures were capped by the $20 \mathrm{~nm}$ thick Si layer formed at temperature $430^{\circ} \mathrm{C}$. It has been shown [13] that germanium quantum dots start to form when the nominal thickness $\left(N_{\mathrm{Ge}}\right)$ of the germanium layer exceeds $4 \mathrm{ML}$. In our experiments we investigated undoped structures with $N_{\mathrm{Ge}}=6$, 8,10 , and $20 \mathrm{ML}$. The planar density of the quantum dots in structures grown in 
similar conditions was approximately $10^{11} \mathrm{~cm}^{-2}$. From the AFM measurements [1-5], which were performed on the samples without capped layers, it results that the height of the quantum dots is about $3 \mathrm{~nm}$, while the lateral size of their basis increases from 7 to $30 \mathrm{~nm}$ on increasing the $N_{\mathrm{Ge}}$ from 6 to $25 \mathrm{ML}$. Beginning from approximately $N_{\mathrm{Ge}}=12 \mathrm{ML}$, relaxation of the elastic deformation takes place by introducing dislocations in the structures.

X-ray absorption near edge structure (XANES) and extended X-ray absorption fine structure (EXAFS) spectra were measured at room temperature at the A1 station in HASYLAB equipped with double crystal Si(111) monochromator. The experiments were done at the $K$-edge of Ge using five-element Ge fluorescence detector. The angles between sample surface and X-ray beam were about $20^{\circ}$ and $75^{\circ}$. Therefore, taking into account the polarization effects one can study bonds out of sample plane $\left(20^{\circ}\right)$ and in plane $\left(75^{\circ}\right)$. The powdered monocrystal of Ge was measured in transmission mode as a reference.

\section{Results and discussion}

In Figs. 1-4 the X-ray absorption spectra of Ge $K$-edge from layers with thickness of 6-20 monolayers buried in $\mathrm{Si}$ and from the Ge reference crystal are collected. All spectra are normalized to the same intensity before the edge and at the end of the spectra.

Considerable changes in spectra intensity and shape for the layers with different thickness, measured at two orientations with respect to the polarization vector of the radiation, were observed. These changes indicate the modification of the morphology of the formed layers with increase in the layer thickness in the considered directions. Comparing spectra for out-of-plane bonds (collected under grazing incidence $\left(20^{\circ}\right)$ and marked in Figs. 1-4 by open circles) for samples with 6 to $20 \mathrm{ML}$, one can notice at first the large increase in X-ray absorption spectroscopy (XAS) intensity between 6 and 8 ML samples and then a continuous decrease in XAS intensity going from 8 to $20 \mathrm{ML}$ samples. Considering the in-plane spectra (collected under normal incidence $\left(75^{\circ}\right)$ and marked in Figs. 1-4 by $\Delta$ ), one can see that the intensity of spectra does not change much for samples with 6 and $8 \mathrm{ML}$ and decreases starting from the sample with $10 \mathrm{ML}$. For the sample with $20 \mathrm{ML}$ the intensity and shape of the spectrum is very close to that for crystalline Ge. Therefore, the atomic arrangement and resulting in-plane electron states distribution for sample with $20 \mathrm{ML}$ of Ge buried in $\mathrm{Si}$ is very close to atomic arrangement in crystalline Ge. Knowing that the lateral size of dots formed in this kind of layer is about $30 \mathrm{~nm}$ [13] our measurements indicate that the in-plane electronic structure for dots of that size is very similar to that for crystalline Ge. The lateral size of dots formed in layers with 6 and $8 \mathrm{ML}$ has the same order of value. Starting from $10 \mathrm{ML}$ the size of dots increases leading to formation of electronic-states distribution similar to crystalline Ge at $20 \mathrm{ML}$. 


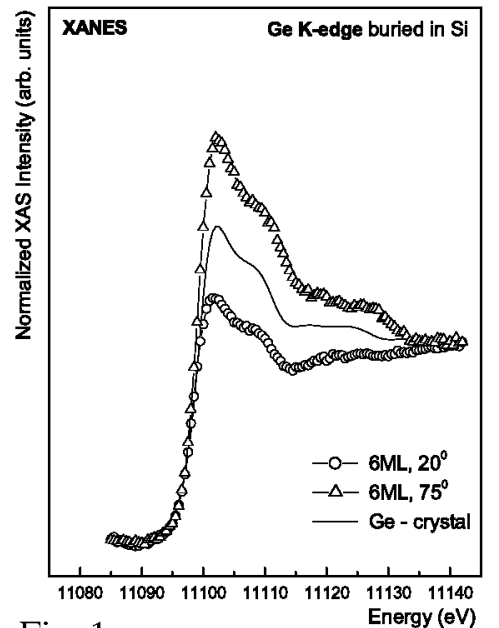

Fig. 1

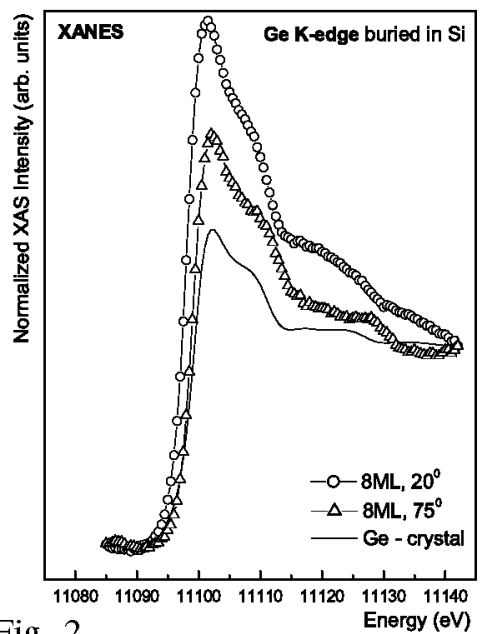

Fig. 2

Fig. 1. XANES of Ge $K$-edge of the crystalline Ge (solid line) and of the sample with Ge $6 \mathrm{ML}$ collected under normal $\left(\Delta-75^{\circ}\right)$ and grazing $\left(0-20^{\circ}\right)$ incidence beam angle.

Fig. 2. XANES of Ge $K$-edge of the crystalline Ge (solid line) and of the sample with Ge $8 \mathrm{ML}$ collected under normal $\left(\Delta-75^{\circ}\right)$ and grazing $\left(0-20^{\circ}\right)$ incidence beam angle.

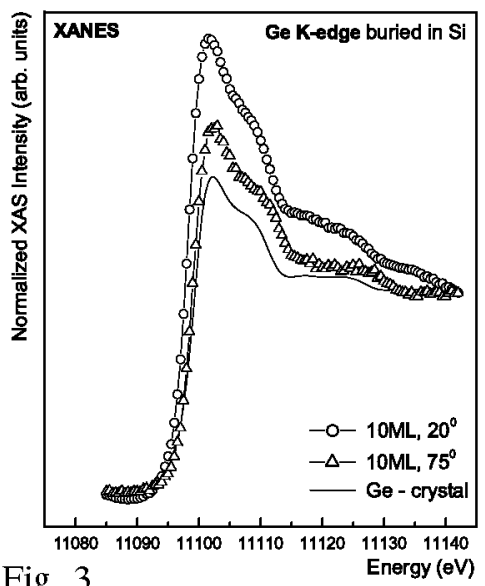

Fig. 3

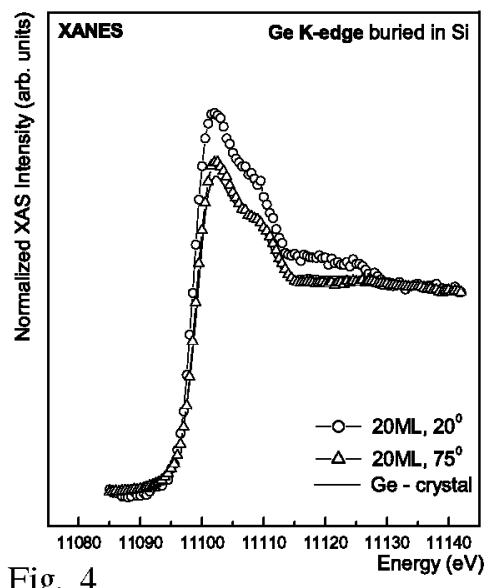

Fig. 4

Fig. 3. XANES of Ge $K$-edge of the crystalline Ge (solid line) and of the sample with Ge $10 \mathrm{ML}$ collected under normal $\left(\Delta-75^{\circ}\right)$ and grazing $\left(0-20^{\circ}\right)$ incidence beam angle.

Fig. 4. XANES of Ge $K$-edge of the crystalline Ge (solid line) and of the sample with Ge $20 \mathrm{ML}$ collected under normal $\left(\Delta-75^{\circ}\right)$ and grazing $\left(0-20^{\circ}\right)$ incidence beam angle. 
The substantial raise of intensity and broadening of the out-of-plane spectra for 8 ML (Fig. 2) can be related to the raise of density of electron states, which is caused by increase in the localization of the states due to potential appearing at the Ge island boundaries. Mixing of the $\mathrm{Si}$ atoms and Ge atoms out-of-plane as well as in-plane of the $8 \mathrm{ML}$ sample took place. In the out-of-plane spectra for $8 \mathrm{ML}$ and $10 \mathrm{ML}$ we observed a chemical shift of the absorption edge which can be related to a change of the ionicity of formed chemical bond and/or of the coordination around Ge atoms (Figs. 2, 3).

Based on the above observations we suppose that, indeed as it was reported [1-5], the formation of hut-like small quantum dots takes place for the layers with thickness from 6 to 10 monolayers of Ge buried in $\mathrm{Si}$ crystals. This particular shape of quantum dots influences the conduction-band structure. The sample with $20 \mathrm{ML}$ Ge has already an in-plane coordination very similar to crystalline Ge, but for the out-of-plane one the differences are still observed that can indicate formation of the dome-like large quantum dots. These dots have the electron states in-plane distribution similar to that for crystalline Ge but the presence of Si atoms in the vicinity of Ge atoms modifies the out-of-plane electronic structure.

EXAFS spectra were measured for the same geometry as XANES ones. A standard EXAFS (without fitting) analysis was done. The pre-edge region was approximated by the linear function for $8 \mathrm{ML}$ - both angles, $10 \mathrm{ML}$ - angle $20^{\circ}$, $20 \mathrm{ML}$ - angle $75^{\circ}$, victorian function for $10 \mathrm{ML}$ - angle $75^{\circ}$ and polynomial function for $20 \mathrm{ML}$ - angle $20^{\circ}$ and Ge crystal. The isolated atom contribution was removed using the spline function. The obtained normalized EXAFS oscillations, $\chi(k)$, multiplied by internal photoelectron wave vector $k^{1}$ for all investigated samples are shown in Figs. 5, 7 and 9 (marked by lines with solid triangles (circles)). As a next step, Fourier transformation (FT) was done using for all samples the Hamming filtering function. The chosen ranges of $k$ for the FT (dependent on a quality of the registered spectra) are listed in Table. The $\chi(k)$ oscillations after filtering are marked by a solid line in Figs. 5, 7 and 9 .

The radial distribution $(R)$ of neighbour atoms around Ge is shown in Figs. 6, 8 , and 10 (in-plane $-75^{\circ}$ and out-of-plane $-20^{\circ}$ ). Considering the shape of radial distribution for out-of-plane case in all investigated samples we can notice that first three coordination shells (up to $\approx 6 \AA$ ) match in position with the crystalline Ge but differ considerably in amplitude indicating the presence of many defects in occupancy of atomic sites. All further shells have different locations and amplitudes with respect to Ge-crystal. For the sample with 20 ML (Fig. 10) the first coordination sphere is shifted in the direction of shorter $R$ (the radial coordinate) and is much broader than for other samples. This indicates the presence of the two shells with different bond length, one $\mathrm{Ge}-\mathrm{Ge}$ and other $\mathrm{Ge}-\mathrm{Si}$, that is in agreement with our conclusion drawn from XANES spectra. 


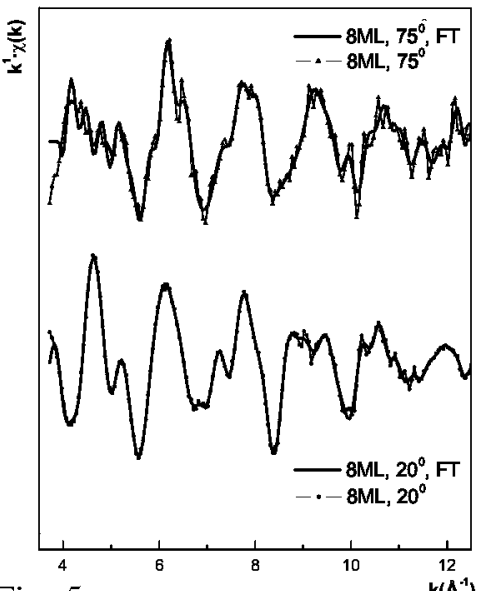

Fig. 5

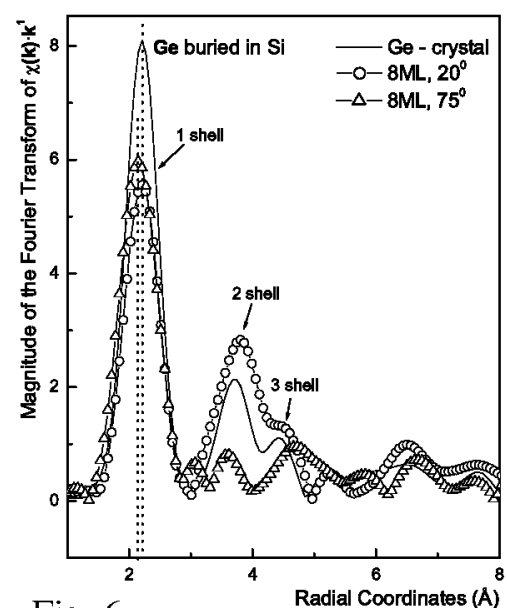

Fig. 6

Fig. 5. Weighted EXAFS oscillations $k \cdot \chi(k)$ from the sample with 8 ML (upper curves for normal incidence $-75^{\circ}$, bottom curves for grazing incidence $-20^{\circ}$ ). Solid triangles and circles — oscillations as measured, the solid line — after filtering.

Fig. 6. The radial distribution function of atoms around Ge in $8 \mathrm{ML}$ sample, after Fourier transformation in the $k$ range specified in Table $(0-$ out of plane and $\Delta-$ in plane), the solid line - in crystalline Ge.

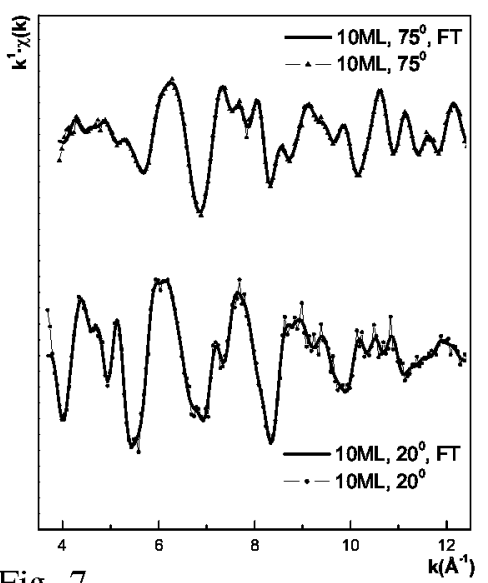

Fig. 7

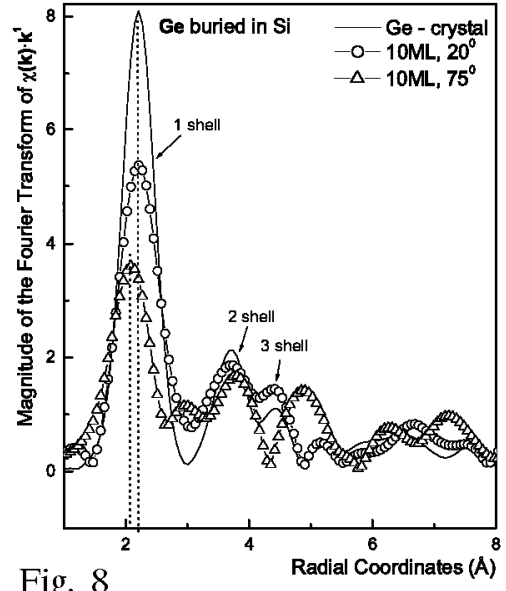

Fig. 8

Fig. 7. Weighted EXAFS oscillations $k \cdot \chi(k)$ from the sample with 10 ML (upper curves for normal incidence $-75^{\circ}$, bottom curves for grazing incidence $-20^{\circ}$ ). Solid triangles and circles - oscillations as measured, the solid line - after filtering.

Fig. 8. The radial distribution function of atoms around Ge in $10 \mathrm{ML}$ sample, after Fourier transformation in the $k$ range specified in Table ( 0 - out of plane and $\Delta-$ in plane), the solid line - in crystalline Ge. 


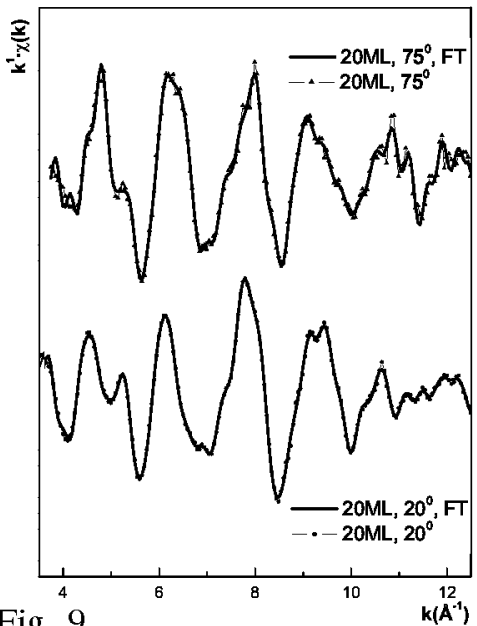

Fig. 9

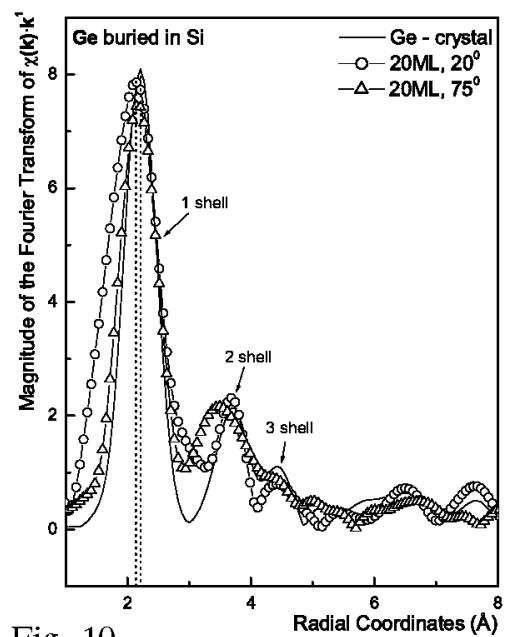

Fig. 10

Fig. 9. Weighted EXAFS oscillations $k \cdot \chi(k)$ from the sample with 20 ML (upper curves for normal incidence $-75^{\circ}$, bottom curves for grazing incidence $-20^{\circ}$ ). Solid triangles and circles - oscillations as measured, the solid line - after filtering.

Fig. 10. The radial distribution function of atoms around Ge in $20 \mathrm{ML}$ sample, after Fourier transformation in the $k$ range specified in Table (o - out of plane and $\Delta-$ in plane), the solid line - in crystalline Ge.

TABLE

The range of $k$ vector chosen for Fourier transformation.

\begin{tabular}{r|c|c}
\hline \hline Sample & $k_{\min }\left[\AA^{-1}\right]$ & $k_{\max }\left[\AA^{-1}\right]$ \\
\hline $8 \mathrm{ML}\left(20^{\circ}\right)$ & 3.80 & 13.5 \\
$8 \mathrm{ML}\left(75^{\circ}\right)$ & 3.95 & 13.3 \\
$10 \mathrm{ML}\left(20^{\circ}\right)$ & 3.85 & 12.95 \\
$10 \mathrm{ML}\left(75^{\circ}\right)$ & 4.0 & 12.7 \\
$20 \mathrm{ML}\left(20^{\circ}\right)$ & 3.3 & 13.5 \\
$20 \mathrm{ML}\left(75^{\circ}\right)$ & 3.7 & 12.4 \\
Ge crystalline & 3.5 & 14.55
\end{tabular}

Considering the radial in-plane distribution we can see that for $8 \mathrm{ML}$ and $10 \mathrm{ML}$ the first shell is shifted in direction of lower $R$ in comparison to crystalline Ge. For 8 ML sample the intensity of the first shell peak is lower than for crystalline Ge, but similar in both considered directions. This is not observed for $10 \mathrm{ML}$ sample, where the first in-plane shell is lower than out-of-plane one, giving an indication of the presence of much more defects in occupancy of atomic sites in plane than out of plane. 
For 20 ML sample the intensity of first-shell peak is similar to that of crystalline Ge in both directions. Moreover, some similarity for 2 and 3 shells with crystalline Ge can be also noticed (Fig. 10) but the second in-plane shell is much broader indicating the presence of two kinds of atoms (Ge and $\mathrm{Si}$ ) and two values of interatomic distances. For the out-of-plane direction, we do not observe this kind of broadening for the second shell.

Summarizing the results of EXAFS analysis one can say that the local order around the Ge atoms in $8 \mathrm{ML}$ and $10 \mathrm{ML}$ samples (out-of-plane) up to the third shell $(\approx 6 \AA)$ is similar to the crystalline Ge. The changes in scattering amplitude indicate the presence of many defects in occupancy of Ge crystalline sites but the atoms are located at the same distances as in crystalline Ge. The mixing of Ge and Si atoms takes place for further shells. In plane only the first shell has similar distance of the neighbour atoms but a shorter bond length than in crystalline Ge. Therefore, the size of the formed dots is smaller in plane than out of plane, or the mixing of Ge and Si atoms already appears in the second shell. In the case of $20 \mathrm{ML}$ sample, in both considered directions a similarity with Ge structure up to the third coordination sphere was observed. Two shells of atoms are clearly seen in the first coordination sphere out of plane. In plane, two subshells can be noticed in second coordination shell and only a very small amount of Si can be admixed to the first shell.

\section{Conclusion}

Standard XANES and EXAFS qualitative analysis of the polarization dependent spectra from layers of Ge with different thickness, buried in Si, was performed. This analysis proves that $\mathrm{X}$-ray absorption spectra are very sensitive to the local order in formed structures and are sources of unique information about the morphology of buried Ge layers. Using these techniques we were able to observe the changes in atomic order around the Ge atoms in investigated buried layers and to compare the formed order with that in crystalline Ge. To draw out more quantitative information, building of models and fitting to the experimental data is necessary. To reduce the thermal disorder and to get a better quality of the experimental data low temperature measurements on the same samples are anticipated.

\section{Acknowledgment}

This work was partially supported by the State Committee for Scientific Research (Poland) grant No. SPUB-M/DESY/P-03/ DZ-213/2000 and by the IHP-Contract HPRI-CT-1999-00040 of the European Commission and Russian Fund for Basic Research (RFBR) grant number is 00-02-18012a under support of which we grew the studied structures. 


\section{References}

[1] G. Capellini, L. Di Gaspare, F. Evangelisti, E. Palange, Appl. Phys. Lett. 70, 493 (1997).

[2] Xun Wang, Zui-min Jiang, Hai-jun Zhu, Appl. Phys. Lett. 71, 3543 (1997).

[3] H. Sunamura, N. Usami, Y. Shiraki, S. Fukatsu, Appl. Phys. Lett. 66, 3024 (1995).

[4] D.J. Eaglesham, F.C. Unterwald, D.C. Jacobson, Phys. Rev. Lett. 70, 966 (1993).

[5] H. Chen, W.G. Cheng, X.G. Xie, Appl. Phys. Lett. 70, 446 (1997).

[6] V.Ya. Aleshkin, N.A. Bekin, N.G. Kalugin, JETP Lett. 67, 48 (1998) (translated from Pis'ma Zh. Eksp. Teor. Fiz. 67, 46 (1998)).

[7] R. Apetz, L. Vescan, A. Hartmann, Appl. Phys. Lett. 66, 445 (1995).

[8] S. Fukatsu, H. Sunamura, Y. Shiraki, S. Komiyama, Appl. Phys. Lett. 71, 258 (1997).

[9] P.D. Persans, P.W. Deelman, K.L. Stokes, Appl. Phys. Lett. 70, 472 (1997).

[10] G. Abstreiter, P. Schittenhelm, C. Engel, Semicond. Sci. Technol. 11, 1521 (1996).

[11] A.B. Talochkin, V.A. Markov, S.P. Suprun, A.I. Nikiforov, JETP Lett. 64, 219 (1996) (translated from Pis'ma Zh. Eksp. Teor. Fiz. 64, 203 (1996)).

[12] P. Castrucci, R. Gunella, M. De Crescenzi, Phys. Rev. B 60, 5759 (1999).

[13] O.A. Shega, K.S. Zhuravlev, V.A. Markov, A.I. Nikiforov, O.P. Pchelyakov, Semiconductors 34, 1311 (2000).

[14] V.A. Markov, H.H. Cheng, Chih-ta Chia, A.I. Nikiforov, V.A. Cherepanov, O.P. Pchelyakov, K.S. Zhuravlev, A.B. Talochkin, E. McGlynn, M.O. Henry, Thin Solid Films 369, 79 (2000).

[15] F. Boscherini, G. Capellini, L. Di Gaspare, F. Rosei, N. Motta, S. Mobilio, Appl. Phys. Lett. 76, 682 (2000). 